\title{
INVESTIGATIVE STUDY OF VOICE AND DATA COMMUNICATION OVER POWER LINE COMMUNICATION SYSTEM
}

\author{
Ganiyu Adedayo Ajenikoko ${ }^{1}$ and Ezekiel Olusegun Ojekunle ${ }^{2}$ \\ ${ }^{1,2}$. Department of Electronic and Electrical Engineering, Ladoke Akintola University of Technology, P.M.B, \\ 4000, Ogbomoso, Nigeria. \\ Corresponding Email: ajeedollar@gmail.com
}

\begin{abstract}
Communication is the transfer of information from one point to another over a channel. However, as technology is growing, more information is needed to be passed over large distances for the realization of the world being a global village. There is the need to place a reliable communication system that will transmit effortlessly both data and voice over a channel. Power Line Communication (PLC) also known as Broadband over Power Line (BPL) technology offers high speed and broadband communication services to homes connected to the power lines. This makes use of the electrical lines for transmission of data up to the last mile and there is no need of separating copper cables, short haul satellite systems, optical fibre cable and Wi-Fi. This work presented an overview of a voice and data communication over PLC in terms of the various types, equipment use, method of communication, application, regulatory activities on PLC and the challenges facing the implementation of power line for transmitting voice and data. Therefore, PLC is a viable alternative to all other methods of transmission as it is readily available and can be easily implemented in rural areas where other communication systems are not implemented for the transmission of voice and data communication.
\end{abstract}

Keywords: Communication, Power Line Communication, Broadband over Power Line, Voice and Data Communication, Broadband over Power Line, Transmission, Electrical Line.

DOI: $10.7176 / \mathrm{MTM} / 9-8-03$

Publication date: August 31st 2019

\subsection{INTRODUCTION}

Over the years, communication has evolved from the Alexander Graham Bell telephone in 1876 to the present Apple iPhone and other smart phones [1]. The idea of just sending audio signals through a cable at a distance has now grown into a global phenomenon [2]. Power Line Communication (PLC) which is the transfer of information using electrical power lines as the communication media or channel has turned out to be a flexible approach to implement low cost and reliable networks in home and industrial environment. This is accomplished by coupling Radio Frequency (RF) energy with Alternating Current (AC) into existing power line using a number of devices at the reception and transmission side $[1,3]$.

PLC as a technology allows the voice and internet data to be transmitted using the existing power lines [4]. Thus, internet service providers can provide additional connections without digging up grounds and erecting poles. In a power plant, devices such as generators, substations, transformers and power lines are used to carry power to the consumers [2]. High voltage (HV) cables are used to carry power from the power station to the substation of different locations using Medium and Low voltage lines (MV and LV). Data cannot be sent 
on high voltage lines due to the large electrical noise generated, instead, the data is transmitted into the medium or low voltage transmission lines. This ensures minimal interference between the power signal and the data signal $[4,5,6]$.

In the voice PLC system, the transmitter sends the voice signal together with an alternating power having the frequency of $50 \mathrm{~Hz}$ [2]. The receiver receives only the voice signal. A power line communication system typically operates by superimposing a modulated carrier frequency signal on the AC signal in a power-line. In-house power-line voice communication is a method of transmitting voice signals at swiftness through a power line in a building, which consists of three basic elements; transmission lines that act as a channel for the transmission of carrier signal, coupling unit that isolates the device from the high voltage transmission line and the transmitter which modulates by adding the voice signal to the AC signal and receivers which demodulates by separating the voice signal from AC signal $[1,3$, $6,7]$.

\subsection{Power Line Communication}

Power Line Communications (PLC) is also known as Power Line Carrier, Power Line Digital Subscriber Line (PDSL), Power Line Telecommunications (PLT) is a novel idea of communication which helps in bridging the gap between the existing electrical and communication network [7]. PLC uses the existing electrical network for communication and allows transmission of data over power lines. PLC works by transmitting high frequency data signals through the same power cable network used for carrying electricity to household users. Such signal cannot pass through a transformer [5]. This requires outdoor devices that combine the voice and data signals with the low voltage supply current in the local transformer stations to bridge the last mile. In the house, indoor devices (adapters) are used in order to filter out the data signals and to feed them to the various applications $[3,8]$.

For communication purpose, reduction in operational costs and expenditures for communication is the main thing in PLC [1]. For internal communication of electrical utilities, PLC is used within buildings and homes (in home PLC) for various communication applications. PLC modems are used to make communication in power supply networks. Data signal from conventional communication devices, (computer, and telephone) is converted by PLC modem in a form that is suitable for transmission over power lines [8]. The PLC transmission channel has some properties such as frequency dependent attenuation, changing impedance, fading and unfavorable noise condition. However, to provide higher data rates, PLC network has to operate in a frequency spectrum of up to $30 \mathrm{MHz}$. PLC network produces electromagnetic radiation and disturb other services operating in the same frequency range [9].

PLC is divided into two groups: narrowband PLC which allows data rates of up to 100 kbps and broadband PLC that allows data rates beyond 2.0 Mbps [8]. With the inevitable arrival of broadband access, the demand for digital voice, video, internet data within the home increases continuously. PLC technology allows the use of existing and widespread power distribution infrastructure to provide high speed networking capabilities along with many other benefits. The PLC network is shown in Figure 1. The PLC networks are connected to the backbone communication networks via a transformer station, or any other station in the network $[10,11,12]$. 


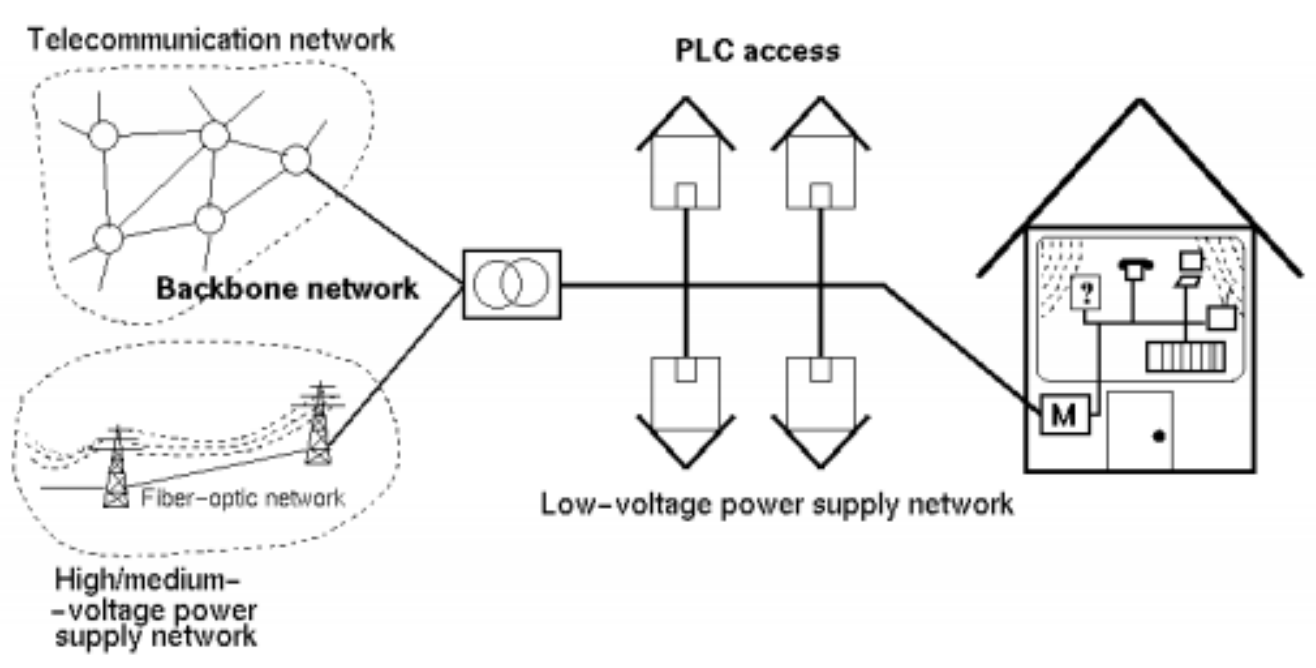

Figure 1: PLC Access Network

\subsection{Applications of Power Line Communication}

PLC also known as Broadband Over power lines (BPL) use existing power lines for streaming data signals (communications). The fundamental principle behind this is that electricity flows over the low-frequency $(50 / 60 \mathrm{Hertz})$ portions of the power lines, leaving room in the higher frequency portions for data streams [6].

Pavlido, et al (2003) stated that the use of PLC technology has been experiencing significant attention lately due to a combination of factors such as the recent advances in solid state integrated circuit technology, the development of improved signal modulation schemes, the liberalization of telecommunications and deregulation of electric utilities. This is also coupled with the birth and growth of the internet and the increasing demand for communication networks for home and office automation, computer communications, security monitoring and several other applications. Hubscher, (2001) also revealed that PLC stems partly from the desire to take advantage of the already extensive network infrastructure of the electric grid, which is estimated to have a worldwide coverage of around $90 \%$. If broadband communication services can be fully achieved using the power distribution network, a truly universal information superhighway with the capability of providing interconnection to every home, office and industry may be realized.

Some special applications of PLC are [10,11, 13]:

1. Automatic Meter Reading (AMR): In this technology, data from energy meter is automatically collected and transfered to the central database for bill and analysis. The main aim for the automation of meter reading is not to reduce labor cost but to obtain data rate that is difficult to obtain. In most of the places, users have demanded that their monthly bill be based on actual reading, instead of the bill which is based on prediction. This is the technology which saves periodic trip and billing is based on the real consumption not estimated. PLC presents an interesting and economical solution for AMR.

2. Home Networking and Internet Access: A large number of computers is connected in a building by using existing network as a Local Area Network (LAN). There is no need to install new wire or cable to connect all the computers due to the availability of low voltage power network which connects all the networks and save the installation cost and time.

3. Home Automation: For remote control of lighting and appliances it is power line communication technique which is used. Power line communication uses existing 
wiring in the home. Transmitting radio programs: Over power line some time power line communication was used for transmitting radio programs. It is known as carrier current system when operated in the Amplitude Modulation (AM) radio band. For communication large portion of the radio spectrum might be used for high frequency communication.

4. Accessibility: Power line communication finds many applications because of its easy accessibility. This communication can be stretched to areas where telephone lines cannot reach. .In advanced future prospects we may be able to enhance the system by providing multiple receivers.

5. Load Control: Many loads may be connected to the output instead of the speaker. These loads may be switches controlling the various appliances or it can be the relays that activate various security systems.

\subsection{VOICE AND DATA COMMUNICATION OVER POWER LINES}

Over the years, the advancement in voice and data communication over power line technology and its potential have led to series of studies to determine its feasibility for use as a communication media in various systems, such as homes, office buildings, cars, airport lighting systems, airplanes and ships that have pre-existing power cables $[6,13,14]$.

In power line voice communication, the module used for transmitting the signal is also used for reception. The Voltage Control Oscillator (VCO) present in Phase Lock Loop (PLL) is used to modulate the signal during transmission [8]. The same PLL is used for demodulating the signal when the module is working as a receiver. Due to this, when the receiver is aligned, the transmitter gets aligned automatically. The speaker used for receiving the audio is also used as the buzzer to alert the user $[5,7]$.

In the receive mode, the mains coupling unit separates the carrier signal present in the main line and passes it to the phase comparator input of the PLL transceiver. Since the free running frequency of the VCO present in the PLL unit is set to the carrier frequency, the frequency deviation in the carrier is reflected as voltage deviation at the output of the PLL unit [9]. This will be the demodulated signal, which is amplified using an audio amplifier, and is fed to the speaker. In the transmitting mode, the signal from the microphone is fed to the VCO of the PLL unit. In addition, the frequency of the VCO is varied with the instantaneous amplitude variation in the Microphone signal and appears at the output. This FM signal is then transmitted to the mains using the coupling unit. The voice and data communication over power-line is shown in Figure $2[14,15]$. 


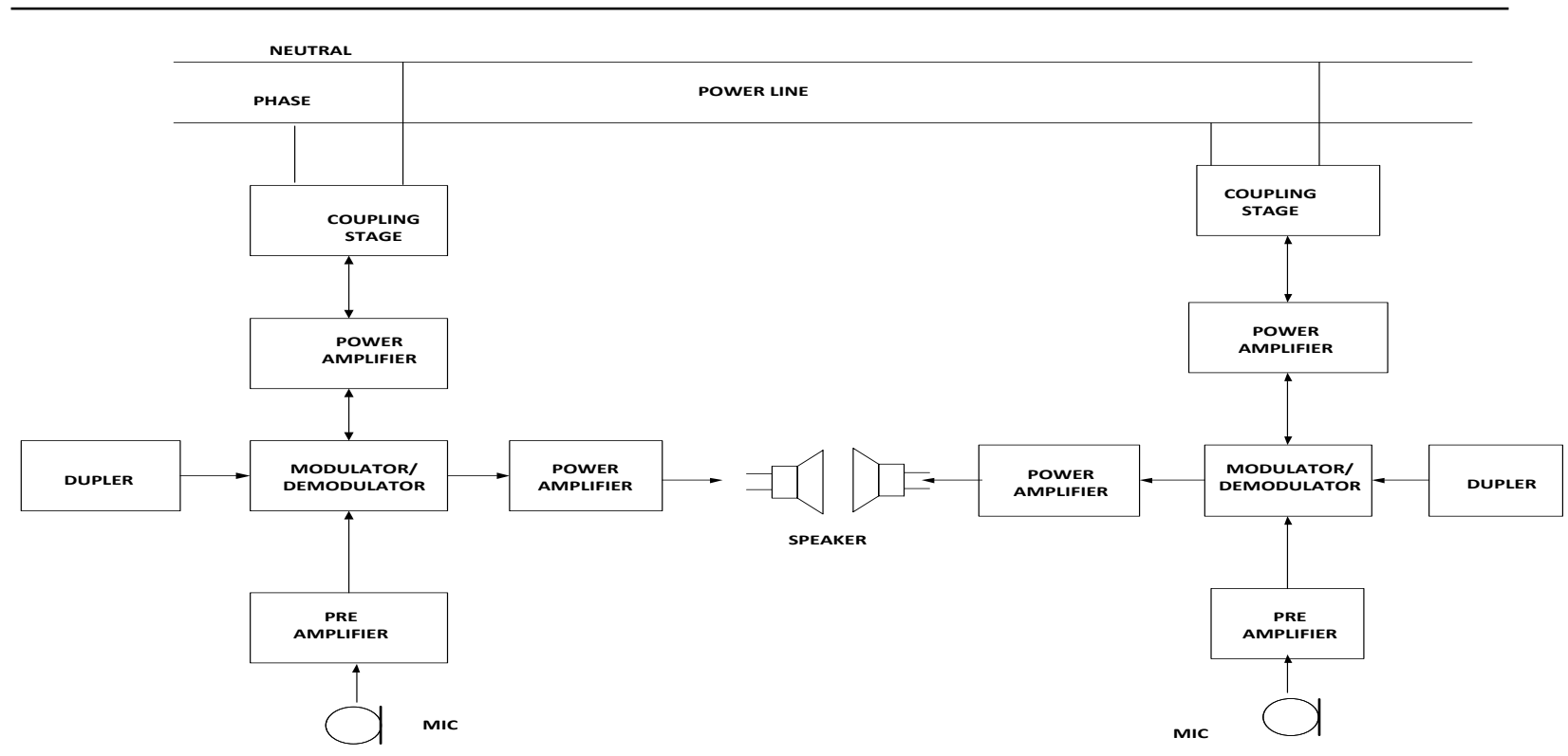

Figure 2: Block Diagram of Voice and Data Communication over Power Line

\subsection{Categories of Voice and Data over Power Line Communication}

Depending on the topology, Power Line Communication (PLC) can be categorized into Access PLC or In-house PLC [3, 16].

\section{A. Access PLC:}

Access PLC is a carrier current system installed and operated on an electric utility service as an unintentional radiator that sends radio frequency energy on frequencies between $1.705 \mathrm{MHz}$ and $80 \mathrm{MHz}$ over medium voltage lines or low voltage lines to provide broadband communications and is located on the supply side of the utility service's points of interconnection with customer premises $[4,16,17]$.

Access PLC provides internet and other broadband services like voice (IP Telephony), video, surveillance systems and entertainment for homes and offices. In this system, injectors are used to provide a connection between internet and the medium voltage power lines. PLC signals can propagate for 1000 to 3000 feet before they become too distorted and weak. To transmit the signals over longer distances, repeaters are used to regenerate and amplify the signal [18]. Extractors are then employed to provide an interface between the users and the medium-voltage power lines. Extractors are typically placed at each distribution transformer which provides low voltage electric power for a group of homes in a particular area $[9,19]$.

Some extractors amplify the PLC signal strength sufficiently to make the transmission of the signal possible through the distribution transformers. Some others employ couplers to bypass distribution transformers and relay the signal to the end-users. Access PLC systems use a combination of medium voltage and low voltage lines or only low voltage lines [20, 21]. The PLC signal is injected onto medium voltage lines and is transferred to low voltage lines using couplers or through low voltage transformers and delivered directly to end users. In the case of low voltage PLC systems, the signal is injected into the low voltage lines at the transformer or the utility meter directly $[15,22]$.

The Access PLC can also be classified as End-to-End Access PLC and Hybrid Access PLC [21, 23, 24, 25]. 
1. End-to-End Access PLC: In this system, a combination of Medium Voltage (MV) and Low Voltage (LV) or LV power lines are either used. The signal is directly injected into the power line and the LV transformer is directly delivered to the end users.

2. Hybrid Access PLC: In the hybrid system, there is a combination of MV power lines and wireless transmission. The signal is injected wirelessly and then using an extractor, the signal is transmitted onto a MV power line which delivers it to the end user. In this scenario the injected PLC signal is sent to the medium voltage lines and is extracted and delivered to the end user by using a wireless channel.

\section{B. In-House PLC}

In-house PLC is a carrier current system, operating as an unintentional radiator, which sends radio frequency energy by conduction over electric power lines that are not owned, operated or controlled by an electric service provider. The electric power lines may be aerial (overhead), underground, or inside the walls, floors or ceilings of user premises. In-house PLC devices may establish closed networks within a user's premises or provide connections to Access PLC networks or both [20, 23, 26, 27].

This application occurs within a building where both ends of the communication link are located. The distance between communication ends is typically less than 100 meters. This system is attractive because no extra wiring is needed within the building. The power lines used belong to the user and not the electricity provider [1,24]. They are connected to the building wiring and uses electrical sockets as their access points. It makes use of a wireless transceiver to access the broadband network [16, 18, 26].

The typical applications of in house power line voice communication are that, this system allows easier and more efficient voice communication between the end users inside a building [28]. In addition, networking and sharing common resources such as printers are other applications of this technology. Figure 3 shows the in-house and Access PLC network [29].

The advantages of using power line as transmission medium for in-house communication are [30, 31]:

1. Affordable and Easy to install: Power-line communication allows user to use their already existing electrical wiring to connect to the different devices. Hence does not require separate wiring for the purpose of communication.

2. Mobility: This system can be helps for occasionally-connected for communication and removed when not in use.

3. Flexibility: Power line communication extends connectivity to all electrical outlets in the home. The same electrical outlets that provide power will also serve as access point for the network devices.

However, the system has some problems that have to be overcome and some aspects that have to be taken into account to realize a successful communication. They are [29, 32]:

1. Minimum-security levels: Power lines do not necessarily provide a secure media because each electrical sink inside a building acts as access point for communication.

2. Voice attenuation: Due to the presence of numerous elements on a power-line network, voice attenuation is likely to be an issue.

3. Noise: The greater amount of electrical noise on the power-line limits practical transmission speed. 


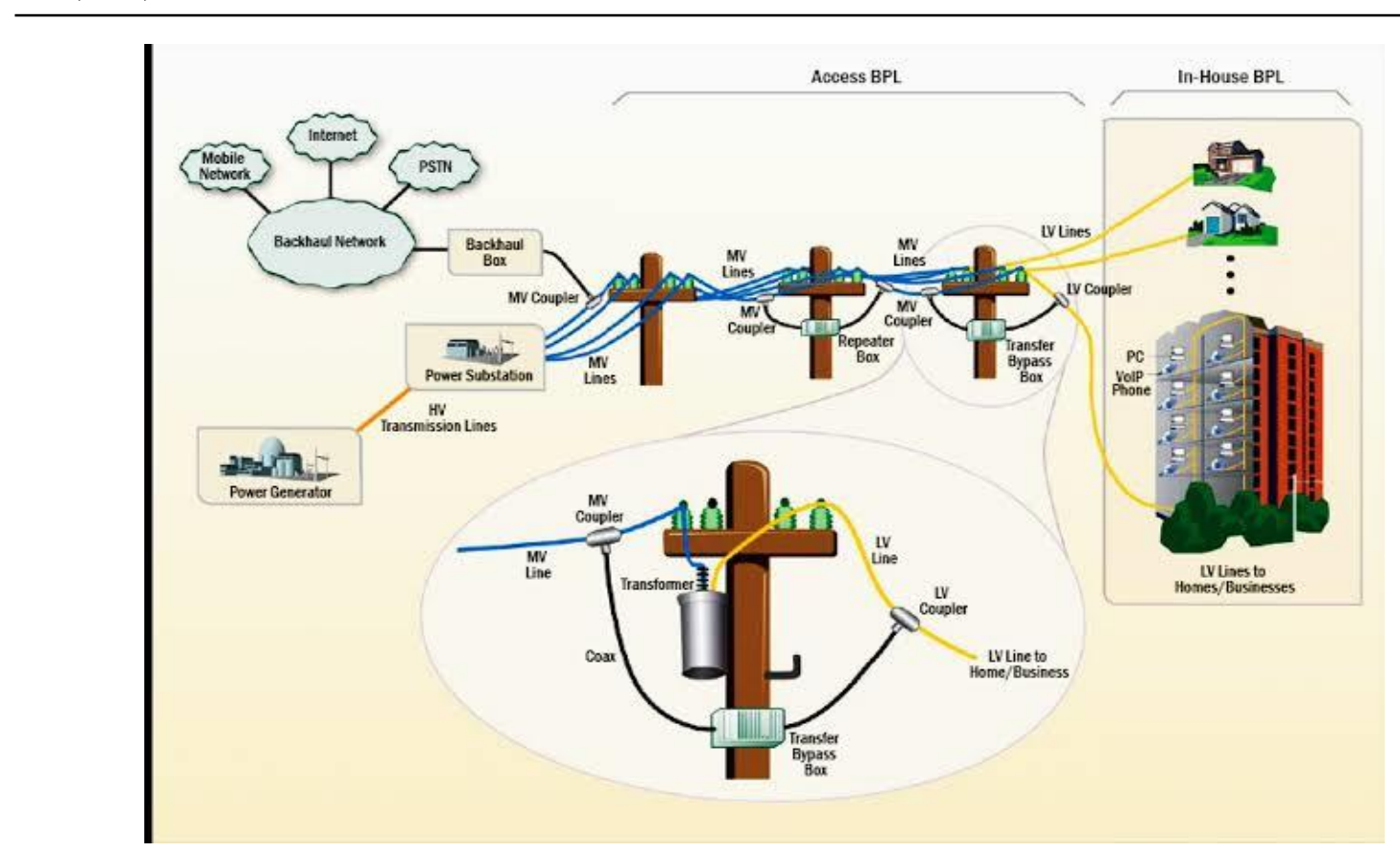

Figure 3: Diagram showing the In-house and Access PLC

\subsection{Equipment used for Voice and Data Communication over Power Line Communications}

There are some equipment which are used in the transmission of data and voice over power lines. These are $[10,19,25]$ :

\section{Microphone and Speaker:}

The Microphone (MIC) contains mouth piece for voice signals. It converts voice signal into suitable electrical signal. In the system design, a condenser MIC is used for voice communication. The speaker converts electrical signal into suitable voice signal. This audio output device is for ring tone alerts at the other module. In this system a 20 watt $4 \mathrm{ohm}$ speaker is used, which have hi-fidelity output handling capacity [22].

\section{Phase Locked Loop Transceiver:}

This Phase Locked Loop block transmits and receives voice signals, hence PLL is bidirectional and controlled by a switching block Duplexer. If duplexer is in receiving mode, ring tone is fed to ring tone generator unit to alert the called party. If called party lifts the hand set, this unit switches to transceiver mode and hence allows both parties to make a conversation $[27,31]$.

\section{Duplexer:}

The duplexer is a multiplexer and acts as digital switch. This change-over-switch is fast enough to change each unit as signal transmitter and receiver, as per the need. When the voice signal is modulated, the Duplexer changes the PLL into transmitting mode and while receiving the voice signal is demodulated and the duplexer changes the PLL into the receiving mode $[26,30]$.

\section{Power Amplifier:}


The audio amplifier boosts caller and called party's audio signals with a gain of 200 . The amplification is necessary as a voice signal has to travel long distance from one module to another. This block is bidirectional as each module receives/transmits voice signals simultaneously. The power amplifier provides the higher current necessary to drive speakers $[28,30,32]$.

\section{Preamplifier:}

A preamplifier is a component normally used with sound equipment to enhance the whole quality of the voice signal. Using preamplifier and the power amplifier, the voice is not changed in quality, but it will be much louder. The preamplifier only supplies a voltage gain $[33,34]$.

\section{Injectors}

Injector is a device which serves as head end to access PLC and it is mounted on a substation. PLC injectors are connected to the internet backbone via fibre-optic or digital signalling level 1 (DS1), or faster, phone lines. The injectors interface to the MV power lines feeding the BPL service area $[5,7]$. MV power lines may be located overhead on utility poles or underground in buried conduit. Overhead wiring is attached to utility poles that are typically 10 meters above the ground. Injectors are used to inject the signals into the medium voltage lines. The internet signal is fed to the input of the injector using a fibre line and medium voltage line connected to the output of the injector. Injectors have three sectors: receiver, signal convertor, and transmitter. Injectors are used to convert the internet signal into a format that can be used to transmit data over power lines [18, 21, 31, 33].

\section{Repeaters}

Repeaters are connected at the poles of the power supply lines. The signal is regenerated at the repeater and is retransmitted. The PLC signal travelling through power lines comes across many channel impairments such as noise, which reduces the bandwidth available to the user $[17,28]$. The repeater has encoder/decoder and regenerator. The repeater decodes the Orthogonal Frequency Division Multiplexing (OFDM) signal, regenerates it and then again converts it to the OFDM signal and transmits it again. The uplink and downlink frequency are kept different to prevent co-channel interference [32, 34].

\section{Extractor}

Extractor is a device used to bypass transformer. An extractor also has a repeater which boosts the signal before transmitting onto power lines $[7,10]$.

\section{Couplers}

Couplers are used to connect the fibre line to medium power line. There are two types of couplers; capacitive and inductive. Capacitive couplers are more efficient as compared to inductive couplers but they require to be physically connected to the electrical lines, which is not the case for inductive couplers. The inductive couplers are connected using ferrite cores [30, 35]. In addition, the use of couplers depends on the voltage carried by the power lines. There are LV couplers that are small in size and can be used with low voltage lines that carry 220 or 110 volts. Furthermore, a coupling unit helps to connect the communication module to the power-line. The purpose of the coupling circuits is to prevent damaging the communication modules by $50 \mathrm{~Hz}, 230$ volts signal used for power supply [31, 34, 36]. 
3.0 METHOD OF VOICE AND DATA COMMUNICATION OVER POWER

\section{LINES}

The general technical idea of Power Line Communication (PLC) is to modulate a radio signal with data and sends it through power lines in a band of frequencies which are not used for supplying electricity [34]. The used frequencies and the modulation scheme have a significant influence on the efficiency and the speed of the PLC service [11]. One of the modulation schemes among others used for voice and data communication over power line communication is Orthogonal Frequency Division Multiplexing (OFDM). This is a multicarrier transmission technique which has been recently recognized as an excellent method for high speed data communication $[35,37,38]$.

Modulation Orthogonal Frequency Division Multiplexing (MOFDM) is a frequency multiplexing scheme utilized as a digital multi carrier modulation method [20]. In this technique, a large number of closely spaced orthogonal sub carriers such as BPSK, QPSK and QAM are used to carry data. The data is further divided into several data channels, one for each sub carrier. Each sub carrier is then modulated with a conventional modulation scheme. Low symbol rate helps in maintaining total data rates similar to conventional modulation schemes in the same range of bandwidth [36]. The orthogonality of sub carriers in MOFDM scheme enables it to achieve distinct advantages over conventional modulation schemes in that it eliminates serious issues of cross talk and interference between sub channels. Besides, inter carrier guards are not required in MOFDM scheme [39, 40].

Differential modulation improves performance in environment where rapid changes in phase are possible. MOFDM can be implemented equally well with coherent modulation and demodulation to maximize the signal to noise ratio $[12,36]$. This approach is preferred for performance-oriented systems, like point to multipoint licensed radios where the highest bit rate per hertz is most important $[38,41]$.

MOFDM technique has acquired added significance in broad band internet access because of its ability to deal with issues of attenuation of high frequencies, narrow band interference and frequency selective fading. The overriding feature of MOFDM is that in this technique, many slowly modulated narrow band signals rather than one rapidly modulated wide band signal is used and this helps in simplification of channel equalization $[1,6]$. MOFDM is generally viewed as a collection of transmission techniques. When applied in wireless environment it is called OFDM. However in a wired environment the term Discrete Multi Tone (DMT) is more commonly used. OFDM is currently used in the European Digital Audio Broadcast (DAB) standards. Several DAB systems proposed for North America are also based on OFDM $[9,31]$. OFDM under the name DMT has also attracted a great deal of attention as an efficient technology for high-speed transmission on the existing telephone networks (e.g. Asymmetric Digital Subscriber Loop or ADSL). A schematic block diagram of OFDM is shown in Figure 4 [34, 42, 43]. 


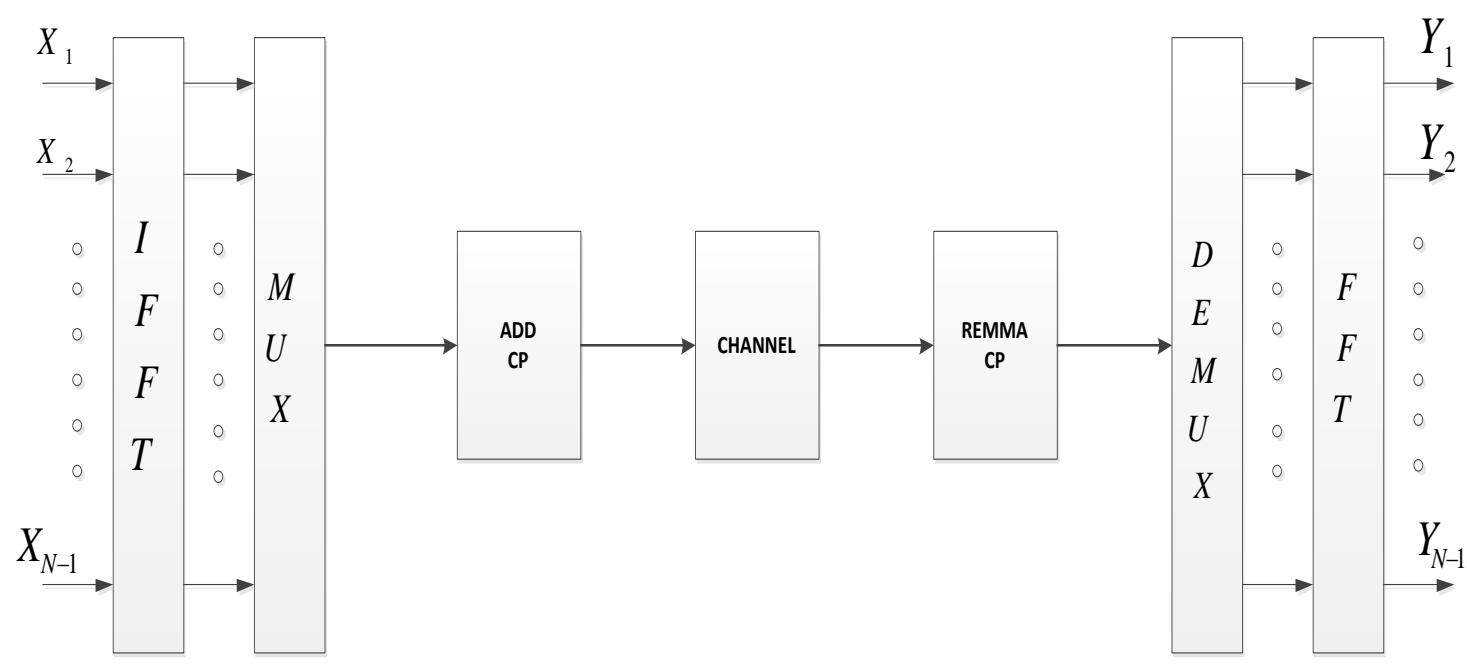

Figure 4: Block Diagram of OFDM System.

The choice of modulation OPDF scheme is dependent on the nature of physical medium on which it has to operate. Modulations OPDF schemes used in power lines have the following desirable properties [1, 44, 45, 46, 47]:

i. Ability to overcome non-linear channel characteristics: Power lines have very nonlinear channel characteristics. This would make equalization very complex and expensive, if not impossible, for data rates above $10 \mathrm{Mbps}$ with single carrier modulation. The modulation technique should have the ability to overcome such nonlinearity without the need for a highly complicated equalization.

ii. Ability to overcome multipath spread: Impedance mismatch on power lines results in echo signal causing delay spread of the order of $1 \mathrm{~ms}$. The modulation technique should have the inherent ability to overcome such multipath effect.

iii. Ability to adjust dynamically: Power line channel characteristics change dynamically as the load on the power supply varies. The technique has the ability to track such changes without involving large overhead or complexity.

iv. Ability to mask certain frequencies: Power line communications equipment use unlicensed frequency band. However it is likely that in the near future various regulatory rules could be developed for these frequency bands also. Hence it is highly desirable to have a modulation technique that could selectively mask certain frequency bands.

In MOFDM, the parallel data streams are first mapped into BPSK data which then modulates a number of subcarriers using discrete Fourier transform (DFT) producing an OFDM signal [5, 38]. An important task in the design of MOFDM systems is the choice of different parameters and the trade-offs between them. An MOFDM symbol starting at $t=t_{s}$, carrying a sequence $d_{i}$ of BPSK symbols in $N$ subcarriers can be expressed by the following complex baseband $[32,48]$ :

$$
\begin{aligned}
& S(t)=\sum_{i=\frac{N}{2}}^{\frac{N}{2}-1} S_{i}+\frac{N}{2} e^{\left\{j 2 \pi \frac{i}{T}\left(t-t_{s}\right)\right\}}, \quad t_{s} \leq t \leq t_{s}+T \\
& S(t)=0, \quad t \leq t_{s} \wedge t>t_{s}+T
\end{aligned}
$$


where; $T$ is the symbol duration.

Here are some of the advantages of MOFDM [33,49, 50]:

i. Very good at mitigating the effects of time dispersion.

ii. Very good at mitigating the effect of in-band narrowband interference.

iii. High bandwidth efficiency.

iv. Scalable to high data rates.

v. Flexible and can be made adaptive; different modulation schemes for sub carriers, bit loading, adaptable bandwidth/data rates possible.

vi. Has excellent ICI performance.

vii. Does not require channel equalization, and phase lock of the local oscillators.

\subsection{Merits and Demerits of Voice and Data over Power Line Communication}

\section{Merits:}

Wide spread and extensive infrastructure that is already available in remote areas in terms of electrical cables allow easy access to internet with relatively very little equipment investment, particularly in areas where limitations in terms of having a cable or DSL connections are experienced by service providers. Maintenance costs of PLC are also extremely low [48]. Cost effectiveness and large scale broadband penetration are two distinct and unique advantages of PLC. In addition, installation time is less than 45 minutes and rural penetration is relatively easy. PLC is a good solution for home networking than other available solutions, as no other infrastructure is required. Access PLC systems have the potential of increasing the availability of broadband services to homes and businesses. PLC systems have been increasing the competitiveness of the broadband services [47, 49]. PLC systems have also been identified as a means of improving the quality and reliability of electric power delivery and creating a more intelligent power grid. PLC technology could allow utilities to more effectively manage power, perform automated metering and monitor the existing power grid for potential failures $[32,50]$.

\section{Demerits}

Power lines are noisy environments for data communications due to several noise sources such as electrical motors, power supplies, fluorescent lights and radio signal interferences. Power line is a shared medium and therefore, the average data rate per end user will be lower than the total capacity depending on coincident utilization, i.e., the number of users on the network at the same time and the applications they are using [50]. Thus, possible technical problems should be comprehensively addressed with various field tests before the PLC technology is widely deployed. In addition, communication over the power lines is lost with devices on the side of an open circuit [41]. This fact severely restricts the usefulness of PLC for applications especially involving switches. In power lines, the attenuation and distortion of signals are immense due to the reasons such as physical topology of the power network and load impedance fluctuation over the power lines. In addition, there is significant signal attenuation at specific frequency bands due to wave reflection at the terminal point [39, $45,46]$.

\subsection{Challenges Facing the Adoption of Voice and Data Communication over Power Line Communication}

A Power Line Communication (PLC) network consists of a wired link and a wireless link. The wired link consists of a pear to pear fiber optical network, with its normal noise and 
attenuation. The wireless portion is mainly affected by all the noises and alternation of free space/atmosphere [40, 44, 47]. Thus, turning on or off of any electrical device every time introduces a click into the line. This becomes quite predominant in case of energy saving devices which introduce quite noisy harmonics into the line [6,7]. The system has to be designed to effectively deal with these natural signaling disruptions. Another major issue is signal strength and operating frequency. The system is expected to use frequencies of 10 to 30 MHZ. Since power lines are unshielded and act as antennas for the signals they carry, they have to interfere with short wave radio frequencies over which PLC operates. This interference becomes quite perceptible in cases where the antennas are physically close to the power lines $[42,46]$.

In the distribution network, the most common interference can be caused by the various house hold appliances and office equipment. The PLC communication system has a transmitter and a receiver with sufficiently low output/input impedance to approximately match channel impedance in the most operating situations. On the other hand, this interference considerably diminishes and is barely perceptible where the antennas are moderately separated from the power network [37, 42, 43].

Some other challenges are $[35,49,50]$ :

1. Radio Frequency (RF) noise interference doesn't interfere with the PLC data signal. PLC signals are in the range of $1.70530 \mathrm{MHz}$ or in some cases $80 \mathrm{MHz}$. PLC uses power lines for providing broadband services which are high frequency alternating current signals are guided through powers lines. These lines act as antennas radiating electromagnetic waves which can possibly hinder other radio communication falling in the same frequency range. This problem can be solved by standardizing frequencies to be used by PLC service providers.

2. Cost: PLC signals operate at high frequency range hence many repeaters may be required to cover large areas. PLC service providers will have to compete with already installed and successful broadband services and has to provide broadband services at competitive rates which is one of the biggest challenges for PLC service providers.

3. Compatibility issues: As there are no standards fixed for PLC service providers, all companies establish different architectures for PLC services hence there are compatibility issues between PLC services in different areas.

\section{A. Noise and Interference in Power Line Communication}

One of the factors which limit the distance of a PLC channel is the noise on the power line, and it must be considered in the design of a PLC channel. Noise is usually composed of randomly occurring voltages, which are unrelated in phase or frequency. Interference on the other hand is usually more structured than noise since it arise as unwanted coupling from just a few signals in the network [12, 23, 48].

The PLC channel must be designed such that the received signal level is greater than the received noise level in the band of the carrier receiver. This depends on the type of modulation and application of the channel [12]. There are two basic types of power line noise; continuous noise and impulse noise. Continuous noise is present at all times and its amplitude is very slow with respect to the frequency considered, while impulse noise exists for only short period of time. The impulse noise has amplitude much greater than the average level of the continuous noise. Both types of noise consist of frequencies that cover the power line carrier band, and considered as white noise over the bandwidth of a carrier receiver [45, 49, 50].

White noise is defined as a noise having a level power density spectrum for all frequencies and an amplitude function which is considered to be random with time. White noise is defined 
as noise having a level power density spectrum for all frequencies and an amplitude function which is considered to be random with time. For the purpose of calculating Signal to Noise Ratio (SNR) and channel performance, the noises is considered to be white noise. The bit error rate of noise is given as $29,48,50]$ :

$$
\begin{aligned}
& B E R=f\left(\frac{E_{b}}{N_{0}}\right) \\
& B E R=0.5 \operatorname{erfc}\left[\sqrt{\frac{\gamma}{2}}\right] \\
& \frac{E_{b}}{N_{0}}=\frac{P_{s} T_{b}}{N_{0}}=\frac{P_{s}}{N_{0}\left(\frac{1}{T_{b}}\right)}
\end{aligned}
$$

Where; $N_{0}$ is the output noise which consists of channel fading, channel noise and interferences, $E_{b}$ is the bit energy, $\gamma$ is the signal to noise ratio (SNR), $P_{s}$ is the signal power, $T_{b}$ is the signal period.

For a power line communication, the noise is assumed to be periodically stationary. So, its mean is zero and variance is synchronous to the $\mathrm{AC}$ voltage of the mains. The probability density function (PDF) of such noise at time $t$ is $[22,32,40]$ :

$$
P(n(t))=\frac{1}{\sqrt{2 \pi \sigma_{n}^{2} t}} e^{\left[\frac{-n^{2} t}{2 \sigma_{n}^{2} t}\right]}
$$

This equation can be taken as a basic equation to model power line noise.

\section{B. Signal Attenuation in Power Line Communication}

Attenuation is the loss of power that a high frequency signal suffers through transmission due to the varied characteristics of the MV lines, LV lines. Various factors affect signal attenuation for access PLC including types of cables used, injection point of PLC signal and injection phase used to inject PLC signal [12, 45, 49].

Signal attenuation occurs more easily on the broadband $(\mathrm{MHz})$ frequency than the narrowband $(\mathrm{KHz})$ frequency [4]. Distribution network simulations carried out on access PLC indicate that signal attenuation increases with increase in frequency. These simulations also indicate that signal attenuation increases with increase in length of power lines. PLC signals over aerial cables experience less attenuation due to lack of dielectric loss than the underground cables. This is also due to the different cable parameters such as total radius [17, $23,41]$.

Bakkali, (2015) indicated that attenuation is low for cables with oilpaper insulation compared to cables with PVC. The type of cables used are significant for full-scale deployment. PLC signal attenuation depends on the position of signal injected. Akarte, (2014) indicated that signal attenuation are more prone when it is injected on to MV wire compared to $\mathrm{LV}$ wire. Attenuation on MV lines is the same for all three phases. 
When the PLC signals reach LV lines, the signal attenuation varies for different phases. This variation is due to the imbalance created by different structures of MV and LV lines. This is a concern for full-scale deployment. Several factors affect signal attenuation for indoor PLC including power line lengths, number of branches, environment and loads connected to the power line network $[5,18,25,48]$.

\section{Interference to other Data Signals}

In PLC systems, modulated data signals are injected into power lines at differential mode. Small portion of these signals escape from the electric wires and interrupt data signals transmitting at the same frequencies by other communication facilities including amateur radios, wireless security services and military surveillance stations. These emissions cause external interference resulting in data loss. In addition, data signals from other communication facilities interfere with PLC signals passing through power lines resulting in data loss $[4,10]$.

PLC signals injected at different mode currents flowing in opposite directions have equal magnitude and phase. The electromagnetic fields generated from the differential mode currents subtract each other. Therefore fields get cancelled out and do not radiate emissions $[8,9]$. These differential mode data signals are converted into common mode current due to untwisted power lines. As the fields get added for common mode currents, unwanted radiations are generated. In addition, power lines transmitted at high frequency emit these electromagnetic radiations due to the antenna behavior of unshielded wires. This asymmetrical power lines causes interference to external signals transmitted at same frequencies $[22,49]$.

In the case of PLC signals, the source is purely resistive component whereas the load is complex with resistive, capacitive and inductive components. Hence source impedance is not equal to load impedance. This mismatch of impedance causes electrical imbalance resulting in asymmetry in power lines, injection points, couplers, power outlets and switches. This asymmetry causes common mode current resulting in emission of electromagnetic radiations which leads to data loss that is not desirable in a good communication system [3, $34,46,50]$.

Various measures include using low power level for BPL signal injection, frequency notching technique, differential mode injection devices, balanced line transmission for access have been used to alleviate interference in PLC. These measures help in reducing interference, which might not completely eliminate interference in the power line network.

\subsection{CONCLUSION}

Power line Communication (PLC) is a technique that allows exchange of data by means of electric power supply network that are presented in every dwelling, office and in every building. In addition, the voice and data communication over power line is not easy to implement as imagined. This research paper has presented an overview of voice and data communication over PLC in terms of the various PLC types, equipment used, method of communication, application, regulatory activities on PLC and the challenges facing the implementation of power line for transmitting voice and data.

In this overview, the evolutions and potentials of PLC technology have been explained. The applications, advantages and disadvantages of PLC technology and some idea of the standards, noise and channel on power line have been discussed. The transmission voice through power line concept has many advantages. The system is inexpensive when compared to other technologies, for example wireless technology for in-house application. 
This system has high potentials in terms of innovations and commercial values due to the uniqueness and effectiveness. PLC is a valid technique that allows the exchange of information by means of power line cables that are present in every dwelling. Information transmitted through power line can be used to share area voice and also to control home and building automation systems. A smart home system improves the independence in the every day's activities, in a comfortable environment which is very personal and peculiar for everyone.

In addition, the study has presented two types of PLC access services; Access PLC and In-House PLC. The Access PLC has many issues at hand. There is interference, cost problem as well as repeater problem. So other access technologies like cable, DSl, 3G are preferred over the Broadband Power Line. The in-house PLC has lesser problems as it distributes the internet using electrical lines in the building. Hence it is an available alternative to the current technology. Furthermore, PLC is a viable alternative to all other methods of transmission as it is readily available and can be easily implemented in rural areas where other communication systems are not implemented for the transmission of voice and data communication.

\subsection{REFERENCES}

[1] Adekunle, O. V. (2012). An overview of broadband communication over power lines. EIE's 118 2nd Intl' Conf.Comp., Energy, Net., Robotics and Telecom, 118-124.

[2] Akarte, V., Punse, N. and Dhanorkar, A. (2014). Power line communication systems. International Journal of Innovative Research in Electrical, Electronics, Instrumentation and Control Engineering, 2(1): 709-713.

[3] Alex, O. (2009). Voice and data communication over power lines. B.Sc Project submitted to Electrical and Information Engineering Department, the University of Nairobi, Kenya, 1-65.

[4] Anastasiadou, D. and Antonakopoulos, T. (2005). Multipath characterization of indoor power-line networks. IEEE Transactions on Power Delivery20 (1), 90-99.

[5] Anatory, J., Mvungi, N. H. and Kissaka, M. M. (2014). Analysis of power line channel model for communication from primary substation node to end-users. Iranian Journal of Electrical and Computer Engineering, 3(1), 9-15.

[6] Anushree, G., Chintan, P., Nadeem, M., \& Tushar, P. (2014, October). Broadband over power lines: internet everywhere. International Journal of Scientific \& Engineering Research, 10, 1018-1022.

[7] Ashcroft, J., Daniels, D. J. and Hart, S. V. (2002). Guide for the selection of communication equipment for emergency first responders. National Institute of Justice, NIJ Guide 104-00; 1-64.

[8] Bakkali, W. (2015). Modeling and optimization of energy consumption for power line communication nsystems. Electric Power, Télécom Bretagne, 1-165.

[9] Bhatia M, and Shamma, R. (2017). A review on power line communication. IEEE Communications Magazine, 4(9): 1-15.

[10] Carlson, A. B., Crilly, P. B. and Rutledge, J. C. (2002). Communication systems, an introduction to signals and noise in electrical communications. McGraw Hill, 2002, 618-620.

[11] Cortés, J. A., Sanz, A., Estopiñán, P. and García, J. I. (2015). Analysis of narrowband power line communication channels f or advanced metering infrastructure. EURASIP Journal on Advances in Signal Processing (2015):27, 1-13.

[12] Fernando, X. N. (n.d.). Broadband over power line: an overview. Research Gate, 1-9. 
[13] Guillen, E. P., López, J. J. and Barahona, C. Y. (2008). Throughput analysis over power line communication channel in an electric noisy scenario. World Academy of Science, Engineering and Technology International Journal of Electronics and Communication Engineering, 2, (7), 1344-1350.

[14] Guzelg“ oz, S., Arslan, H., Islam, A. and Domijan, A. (2011). A review of wireless and plc propagation channel characteristics for smart grid environments. Hindawi Publishing Corporation, Journal of Electrical and Computer Engineering, 2011, Article ID 154040,1-13.

[15] Hashmat, R., Pagani, P., Zeddam, A. and Chonave, T. (2011). A channel model for multiple input multiple output in-home power line networks. IEEE International Symposium on Power Line Communications and Its Application, 35-41.

[16] Hassan, A. (2013). Enhanced in-house voice communication over power line network. International Journal of Scientific and Research Publications, 3(7): 1-5.

[17] Held, G. (2006). Understanding Broadband over Power Line. Boca Raton, Florida: Auerbach Publications.

[18] Hubscher, B. (2001). Making broadband PLC a commercial reality. Modern Power Systems, XXI, 55-58.

[19] ITU-R SM. (2011). Impact of power line telecommunication systems on radio communication systems operating in the VHF and UHF bands above $80 \mathrm{MHz}$. SM Series Spectrum management, 1-72.

[20] Jae, Y. P., Choong, S. H., Sungwon, L. (2011). An efficient MAC scheme with modified RTS/CTS of IEEE P1901. Int. Conference Information Networking (ICOIN), 230-234.

[21] Kouwen, A., Scanlon, M., Choo, K. R. AND Khac, N. A. (2010). Digital forensic investigation of two-way radio communication equipment and services. Forensics and Security Research Group, 1-26.

[22] Lampe, L., Newbury, J., Swart, T. and Ferreira, E. H. (2010). Power line communications. New York, NY: John Wiley \& Sons, 1-26.

[23] Llano, A., Angulo, I., Angueira, P., Arzuaga, T. and Vega, D. (2016). Analysis of the channel influence to power line communications based on ITU-T G.9904 (PRIME). Energies 2016, 9, 39; doi:10.3390/en9010039, 9-39.

[24] Mannan, A., Saxena, D. K. and Banday, M. (2014). A study on power line communication. International Journal of Scientific and Research Publications, 4(7): $1-4$.

[25] Marcuzzi, F. and Tonello, A. M. (2018). Radio access network backhauling using power line communications. Broadband Communications Networks - Recent Advances and Lessons from Practice, 59-75.

[26] Mayank, S., Shiva, S., \& Sushant, R. (2014). Broadband over power line -overview. International Journal of Advanced Research in Electrical, Electronics and Instrumentation Engineering, III(3), 7564-7567.

[27] Oborkhale, L. I. and Shoewu, O. (2007). Power line communication technology. The Pacific Journal of Science and Technology, 8(2) 398-405.

[28] Paul, C. (2008). Analysis of multiconductor transmission lines. 2nd Edition, John Wiley and Sons, New Jersey, 2008.

[29] Pavlido, N., Han Vinck, A., Yazdani, J., \& Honary, B. (2003). Power line communications: state of the art and future trends. IEEE Communications Magazine, $X L, 34-40$.

[30] Probir K. B. (2012). Introduction to radio communication. IEEE communication paper, 4(4): 78-86. 
[31] Rapp, M., Dostert, K. and Gotz, M. (2004). Power line channel characteristics and their effect on communication system design, IEEE Communications Magazine, 42(4): 78-86.

[32] Rashya, B. E. (2014). Design and implementation of efficacies data transfer through power line communication. International Journal of Advanced Research in Electronics and Communication Engineering (IJARECE), 3(11)1628-1631.

[33] Rasi, H. and Gilan, M. S. (2016). New method for data communication over powerline career. International Journal of Advanced Biotechnology and Research (IJBR), 7(5): 993-1002.

[34] Sagar, N. (2011). Power line communications systems: overview and analysis. A thesis submitted to the Graduate School-New Brunswick Rutgers, The State University of New Jersey In partial fulfillment of the requirements For the degree of Master of Science Graduate Program in Electrical and Computer Engineering, New Brunswick, New Jersey, 1-160.

[35] Sartenaer, T. and Delogne, P. (2006). Deterministic modeling of the (shielded) outdoor power line channel based on the multiconductor transmission line equations. IEEE Journal on Selected Areas in Communications 24 (7), 1277-1291.

[36] Serrao, J., Fakih, A. and Khatik, R. (2012). Transmission of data using power line carrier communication system. International Journal of Electronics Communication and Computer Technology (IJECCT), 2(6): 280-283.

[37] Singh, H. R., and Gupta, S. (2016). Broadband communication over power lines: issues, challenges and opportunities. International Journal of Advanced Research in Computer Science, VII(7), 108-119.

[38] Singh, V. P. (2012). Analysis of power line communication channel model using communication techniques. Master of Science in Electrical and Computer Engineering, North Dakota State University, 1-49.

[39] Tang, S., Xie, Y., Tang, C. and Jinwenzhu, G. (2012). A comparative study of power line communication networks with and without Buffer. Int. Conference Information Networking (ICOIN), 4(2): 1-23.

[40] Tonello, A. M. (2007). Wideband impulse modulation and receiver algorithms for multiuser power line communications. EURASIP Journal onAdvances in Signal Processing, 1-16.

[41] Tonello, A. M., Versolatto, F., Bejar, B. and Zazo, S. (2012). A Fitting Algorithm for Random Modeling the PLC Channel. IEEE Transactions onPower Delivery 27 (3) (2012) 1477-1484.

[42] Tsuchiya, F. (2002). Measurements of harmful interference in the HF, UHF bands caused by extension of power line communication bandwidth. Int. Conference Inform. And Commun. Technologies: From Theory to Applications, 21: 1-14.

[43] Varma, M. K., Jaffery, Z. A. and Ibraheem, K. (2019). Broadband power line communication: the channel and noise analysis for a power line network. International Journal of Computer Networks \& Communications (IJCNC), 11(1), 81-92.

[44] Veronesi, D., Riva, R., Bisaglia, P., Osnato, F., Afkhamie, K., Nayagam, A., Rende, D. and Yonge, L. (2011). Characterization of in-home MIMO power line channels. IEEE International Symposium on Power Line Communications and Its Applications, IEEE, 2011, pp. 42-47.

[45] Watson, J. (2018). Broadband over power lines. Retrieved from Wikipedia: https://en.m.wikipedia.org/wiki/Broadband_over_power_lines\#Technical_challenges 
[46] Yin, Y. (2017). Application of PLC Control System and Communication Technology in Chemical Plant Equipment Renovation. Chemical Engineering Transactions, 62, 679-684.

[47] Yousuf, M. S. and El-Shafei, M. (2008). Power Line Communications: An Overview Part I. IEEE, 978-1-4244-1841-1/08; 218-222.

[48] Yousuf, M. S., Rizvi, S. Z. and El-Shafei, M. (2008). Power line communications: an overview-part ii. ICTTA Int. Conference Inform. and Commun. Technologies: From Theory to Applications, 1-6.

[49] Zimmermann, M. and Dostert, K. (2002). A multipath model for the power line channel. IEEE Trans. Commun., (50), 553-559.

[50] Zwane, F. (2014). Power line communication channel modelling. Dissertation Submitted in Fulfilment of the requirements for the degree Master of Science in Engineering: Electronic Engineering in the College of Agriculture, Engineering and Science, University of Kwazulu-Natal, 1-92. 